\title{
Frequency intermittency and energy pumping by linear attachments
}

\author{
N. Roveri ${ }^{\mathrm{a}, *}$, A. Carcaterra $^{\mathrm{a}}$, A. Akay ${ }^{\mathrm{b}}$ \\ a Department of Mechanics and Aeronautics, University of Rome, “La Sapienza”, Via Eudossiana, 18, 00184 Rome, Italy \\ ${ }^{\mathrm{b}}$ Department of Mechanical Engineering, Bilkent University, Ankara 06800, Turkey
}

\section{A R T I C L E I N F O}

\section{Article history:}

Received 12 April 2013

Received in revised form

25 February 2014

Accepted 2 April 2014

Handling Editor: D.J. Wagg

\begin{abstract}
A B S T R A C T
The present paper considers the problem of realizing an effective targeted energy pumping from a linear oscillator to a set of ungrounded linear resonators attached to it. Theoretical as well as numerical results demonstrate the efficacy of using a complex attachment as a passive absorber of broadband energy injected into the primary structure. The paper unveils also the existence of an instantaneous frequency associated with the master response characterized by intermittency: a rather surprising result for a linear autonomous system. Comparison with nonlinear energy sinks demonstrates that the two systems have some analogies in this respect and that the linear complex attachment is a very efficient energy trap.
\end{abstract}

(c) 2014 Elsevier Ltd. All rights reserved.

\section{Introduction}

In the past three decades new methods have been invented and employed for vibrations absorption of stationary and transient responses of many types of engineering structures. Conventional vibration absorbers designed for civil structures are generally linear and require the addition of large masses added to the primary structure, which makes these systems of limited interest. Moreover, they are effective over a narrow frequency range, showing poor performance in case of broadband inputs. To mitigate some of these problems, nonlinear techniques have been extensively studied [1-16].

One such method involves the use of a weakly nonlinear vibration absorber, added to the principal structure, which can operate under various types of external excitation [1-6]. Damping in the absorber plays a fundamental role and governs the effective bandwidth of absorption, introducing a tradeoff between attenuation efficiency and bandwidth.

Strongly nonlinear passive absorbers [2-5] have shown to provide better performance than the corresponding linear and weakly nonlinear devices. These include targeted energy pumping into the nonlinear absorber. Energy pumping describes controlled and irreversible transfer of vibrational energy, from a vibrating main structure to a passive, essentially nonlinear, attachment, where it remains trapped and dissipated. The attachment essentially acts as a nonlinear energy sink (NES); a number of papers have shown that a proper design of the absorbing structure permits a nonlinear mode of vibration, which leads to energy pumping [8-10]. Several studies employ NES with cubic stiffness nonlinearity $[6,7,11,15]$ and in some, the mass of the NES is comparable to that of the primary structure [11,15]. Strong nonlinear attachments are effective over a wider range of frequencies than common linear and weakly nonlinear devices [12-14].

\footnotetext{
* Corresponding author. Tel.: +3906 44585794; fax: + 3906484854.

E-mail address: nicola.roveri@uniroma1.it (N. Roveri).
} 
Despite the important advantages of using NES, they are accompanied by certain drawbacks. One relates to systems containing nonlinearities, namely the existence of additional branches of undesired periodic responses, giving rise to highamplitude excitations of the entire system [51]. There exist certain conditions, i.e. cubic nonlinearity with light quadratic damping, where the vibration induced by the NES may be very high. Another drawback is that energy pumping is activated only above a specific energy value: when the vibratory energy is below a threshold level, NES is not effective [15]. Finally, a small mass for the NES is required for an efficient application to real structures [11,15], while in many examples the mass of the NES is of the same order than the one of the primary.

Research on nonlinear attachments has been focused on finding methods for efficient energy pumping. It is well known that irreversibility, in physical systems with a finite number of degrees of freedom, develops as a consequence of nonlinearities [16,17]. Nonlinearity destroys the periodic motion in linear systems [46], thus the energy remains widely spread among the degrees of freedom and never reorganizes itself to return to its source, giving rise to irreversibility. However, weak nonlinearities are not as effective in producing irreversibility: this result has been empirically confirmed [1-6], and holds in general for Hamiltonian isochronous or non-isochronous systems, as stated by Nekhoroshev's theorem $[18,19]$, or by the complementary and more celebrated Kolmogorov-Arnold-Moser theorem [18,20], respectively. In a nutshell, the theorems show that the motion of a weakly nonlinear system remains very close to that of the corresponding linearized system, without developing irreversibility.

These arguments suggest that irreversibility (i) is a result of particular strong nonlinearities and (ii) does not normally take place in linear systems, because their modal energies are constants of motion. However, the latter notion requires deeper investigations in light of results from a special class of linear systems [21-36,45], which exhibit irreversible energy transfer. A prototypical example of these systems consists of a principal structure, called master, which is a one dimensional resonator, coupled to a large number of parallel resonators, constituting the attachment. The whole system is linear but its special architecture allows a very effective energy pumping, from the principal to the attachment, which acts as a linear energy sink (LES). We refer to the whole system simply as master-attachment, while complex attachment (CoAt) is adopted for the satellite structure.

The energy sharing process in master-attachment systems has been investigated in some detail [21-36], which was, in part, inspired by the fuzzy damping concept introduced by Soize et al. [21]. The prototypical system addressed here relates to many engineering applications with fundamental physical questions. In fact, the schematization of many engineering architectures frequently uses the paradigm of an attachment of a population of resonators to a master system, like in any vehicle analysis where a principal structure, like the car body, the airplane fuselage, or the hull of a ship, are coupled to a very large number of resonating components. The high level of damping experienced in these structures cannot simply be explained by the concept of nonlinear connections. Moreover, many interesting applications of the concept of CoAt can be used for designing novel vibration absorbers [40].

Using a deterministic approach, it has been shown [22-25] that satellite oscillators act as a viscous damper on the master structure, increasing considerably its effective damping. Moreover, the damping induced on the master is independent on the energy dissipation within the attachment. Further, if the number of resonators is high, approaching infinity, even for vanishing values of loss factor in each oscillator the satellite structure acts as a viscous damper on the master. Weaver, in a series of investigations [26,27], generally corroborates these results, providing alternative approaches. In the case of a finite number of resonators, the damping behavior of the attachment holds true only for early times. There exists a return time $t^{*}$ after which the energy is returned to the master and the satellite structure stops acting as a viscous damper. On this basis the term apparent damping has been used to describe the complete energy transfer from the master to the attachment which takes place up to the time $t^{*}$. Thus, the time behavior of a finite structure is qualitatively different of the one of an infinite structure after $t^{*}$. This is consistent with the notion that in linear undamped systems energy transfer from the master to the satellite oscillators is reversible.

A related issue concerns the definition of apparent damping compared to true dissipation. The problem was tackled by several authors: Langley [29], Celik and Akay [30], Strasberg [24] and Maidanik [28]. Particularly, Maidanik showed linear systems require a physical loss mechanism for the energy to be actually dissipated. If the resonators of the attachment have zero loss, as long as their number is finite, no matter how large, the resulting damping is only apparent and no energy is dissipated.

A generalization of the estimate of the return time $t^{*}$ has been provided by Carcaterra and Akay [31]. They showed that $t^{*}$ can be modified with the frequency distribution within the set of oscillators, i.e. $t^{*}$ depends on the number of degrees of freedom $N$ : the higher the $N$ the longer the $t^{*}[31-37,45]$. A family of special frequency distributions was shown to be able to trap the energy within a linear attachment very effectively [35,36].

In spite of the rich literature on the subject, there still exist a number of issues related to the use of a CoAt for targeted energy transfer that deserve to be further investigated. The attention, in the early studies, has been mainly focused on the early stage of the energy sharing process. However, in later stages, the motion of the master undergoes a transition, from an almost-periodic to a random-like motion, a process that has not been completely understood. Other important issues that arise from these arguments include whether apparent damping is actually an irreversible phenomenon.

The purpose of this paper is to provide insight into these open questions. In this work we present theoretical and numerical evidences of energy pumping employing LES. The basic phenomenology of a CoAt is introduced in Section 2, where a comparative study with an essentially nonlinear attachment is also provided, in Section 2.1. In Section 3, a theoretical model describing the mechanism behind apparent damping is proposed, which predicts the existence of an 
instantaneous frequency associated with the master response characterized by intermittency. These findings are validated numerically, using empirical mode decomposition and normalized Hilbert transform, which provide the empirical evidence of the intermittent frequency. Section 4 contains concluding remarks.

\section{Basic phenomenology of linear energy sink}

The prototype linear system representing a one-dimensional oscillator coupled to a set of parallel oscillators is depicted in Fig. 1. The complex attachment is characterized by a large number of resonators, typically more than 100; the word complex is used to underscore the collective behavior of a large number of independent resonators within the satellite. The total mass of the attachment is a small fraction that the master, typically $1 / 10$.

Results show that as the primary structure is excited, the injected energy $E_{\text {tot }}$ flows quickly to the CoAt where nearly all the energy remains stored up to time $t^{*}[26,31]$. After $t^{*}$ most of the energy returns to the master. This phenomenon is called the apparent damping because, although energy dissipation is not involved in this conservative system, the master reacts as a damped resonator up to $t^{*}$. Typical time history of the master displacement is shown in Fig. 2.

Observing the phenomena in the long time range $\left(t^{*}<t<30 t^{*}\right)$, different energy sharing processes are displayed in Fig. 3: (i) at early stage in this period energy is periodically transferred to and from the primary, (ii) peak values of the energy progressively decrease and (iii) the periodicity of the energy transfer gradually vanishes, (iv) the motion of the master approaches a random noise about a mean value and ( $v$ ) nearly all the energy remains trapped within the CoAt.

The energy exchange of the prototypical system described here is substantially independent of the intrinsic damping [22-26], i.e. of any actual dissipation mechanism, as long as it is very low. When a small amount of dissipation is included, differences between responses of conservative and dissipative master-attachment systems become appreciable only in long times, when the energy of the damped system tends to zero. For a qualitative study of the phenomenon dissipation is not taken into account: in this case, these systems belong to a class of Hamiltonian systems [43,44,46-49].

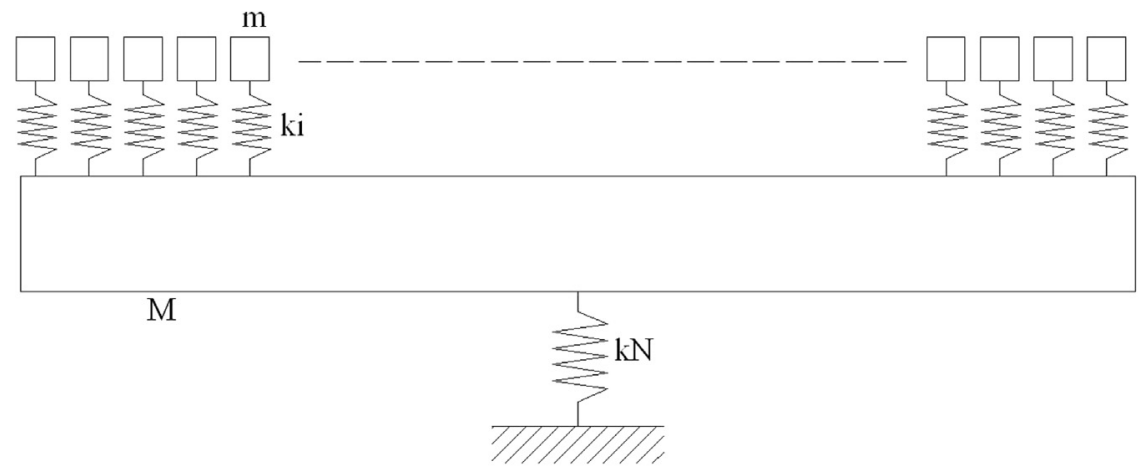

Fig. 1. Prototype of master-attachment system.

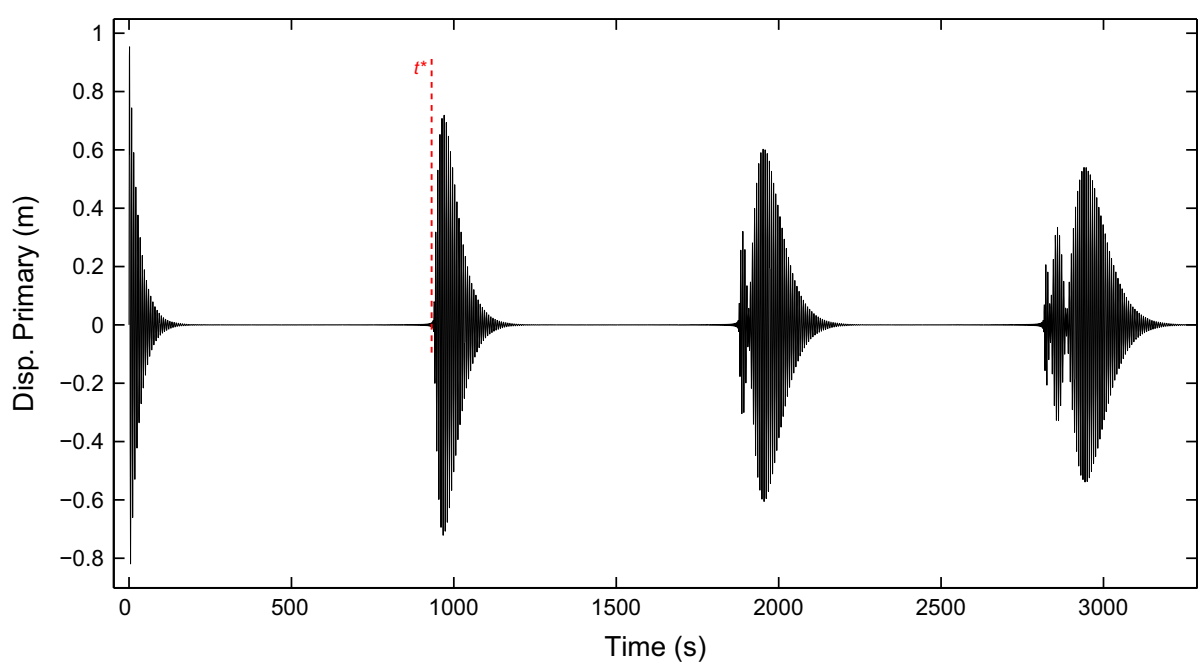

Fig. 2. Displacement of the master versus time, for an impulsive excitation of the master with $E_{\text {tot }}=0.5 \mathrm{~J}$. 


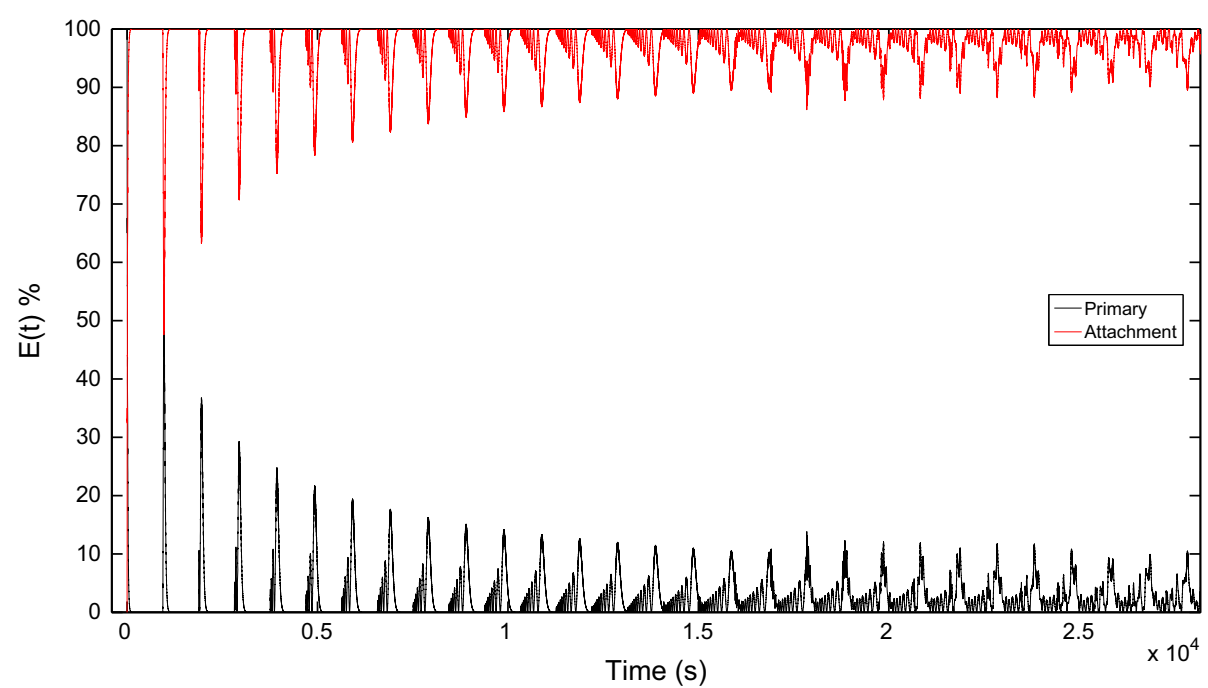

Fig. 3. Percentage of the instantaneous time energy within the master and the CoAt, where $E_{\text {attach }}(t)=\left(1-E_{N}(t) / E_{\text {tot }}\right)$, plotted versus time, for a linear frequency distribution.

The free vibrations of a master-attachment system with $N$ degrees of freedom are described by the equations:

$$
\left\{\begin{array}{l}
m \ddot{x}_{j}(t)+k_{j}\left(x_{j}(t)-x_{N}(t)\right)=0 \quad j=1,2, \ldots, N-1 \\
M \ddot{x}_{N}(t)+k_{N} x_{N}(t)+\sum_{j=1}^{N-1} k_{j}\left(x_{N}(t)-x_{j}(t)\right)=0
\end{array}\right.
$$

where the index $N$ is for the master and $1,2, \ldots, N-1$ for the oscillators of the attachment; $m$ is the uniform mass of each oscillator of the attachment, $k_{j}, M, k_{N}$, and $x_{j}(t)$ are the stiffness of each oscillator of the attachment, the mass and the stiffness of the master and the displacement of the $j$-th oscillator, respectively.

The apparent damping effect develops only when the master frequency $\omega_{M}=\sqrt{k_{N} / M}$ belongs to the frequency interval $B=\left[\omega_{01}, \omega_{0 N-1}\right]$ described by the uncoupled frequencies of the oscillators within the attachment, i.e. $\omega_{0 i}=\sqrt{k_{i} / m}$. When the master frequency lies outside $B$, the satellite acts roughly as a one dimensional, linear, resonator and is not able to efficiently trap the energy.

From Eq. (1), choosing as initial conditions an impulse of velocity $V_{0}$ acting on the master, i.e. $\mathbf{x}(0)=\mathbf{0}$, $\dot{\mathbf{x}}(0)=\left(0, \ldots, 0, V_{0}\right)^{T}$, using modal analysis, the displacement and the velocity of each resonator can be expressed as

$$
\begin{array}{r}
x_{i}(t)=\sum_{j=1}^{N} \omega_{j}^{-1} U_{i j} \sqrt{2 E_{j}} \sin \left(\omega_{j} t\right) \\
\dot{x}_{i}(t)=\sum_{j=1}^{N} U_{i j} \sqrt{2 E_{j}} \cos \left(\omega_{j} t\right)
\end{array}
$$

where $\omega_{j}, U_{i j}$ and $E_{j}$ are the eigenvalues the eigenvectors and the modal energies of the system, respectively

$$
E_{j}=M E_{\text {tot }} U_{N_{j}}^{2}
$$

with $E_{\mathrm{tot}}=M V_{0}^{2} / 2$. From Eq. (2), the displacement of the primary structure is rewritten as follows:

$$
x_{N}(t)=\sum_{j=1}^{N} a_{j} \sin \left(\omega_{j} t\right)
$$

where $a_{j}=V_{0} E_{j} /\left(\omega_{j} E_{\text {tot }}\right)$.

The energy of the master is defined as

$$
E_{N}(t)=0.5 M\left(\dot{x}_{N}^{2}(t)+\omega_{M}^{2} x_{N}^{2}(t)\right)
$$

The energy ratios $E_{N}(t) / E_{\text {tot }}$ and $E_{\text {attach }}(t)=\left(1-E_{N}(t) / E_{\text {tot }}\right)$ are used as indicators of the energy distribution among the resonators, as illustrated in Fig. 3.

Substituting Eq. (3) into Eq. (4), the energy of the master becomes

$$
E_{N}(t)=\sum_{j=1}^{N} \sum_{q=1}^{N} \frac{E_{j} E_{q}}{E_{\text {tot }}}\left(\cos \varphi_{j}(t) \cos \varphi_{q}(t)+\frac{\omega_{M}^{2}}{\omega_{j} \omega_{q}} \sin \varphi_{j}(t) \sin \varphi_{q}(t)\right)
$$


where $\varphi_{j}(t)=\omega_{j} t$ is the phase-angle. Under the hypothesis that the bandwidth $B$ is small enough, such that $\omega_{M}^{2} / \omega_{j} \omega_{q} \approx 1, \forall j, q$, the nondimensional energy becomes

$$
\varepsilon(t) \approx\left[\varepsilon_{N}\right]+\sum_{j \neq q} \varepsilon_{j} \varepsilon_{q} \cos \left(\omega_{j}-\omega_{q}\right) t
$$

where $\varepsilon_{j}=E_{j} / E_{\text {tot }}$ is the normalized modal energy. It is possible to show [36] that

$$
\left[\varepsilon_{N}\right]=\lim _{t \rightarrow \infty} \frac{1}{T} \int_{0}^{T} \frac{E_{N}(t)}{E_{\mathrm{tot}}} \mathrm{d} t \approx \frac{1}{N}
$$

As shown in Fig. 3, the energy peaks are progressively less energetic and, after an initial transient, they remain close to the mean value (7), which can be made negligibly small by simply increasing the number of resonators. The complexity of the satellite plays a fundamental role in producing effective energy storage within the attachment: through a proper selection of $N$ it is possible to select the residual energy that remains with the master, while keeping the mass of the satellite unchanged.

Fig. 3 also shows the energy fluxes between the primary and the CoAt, equipped with equispaced frequencies, i.e. $\omega_{0 i}=i 2 \omega_{M} /(N-1)$ within the bandwidth $B=\left[\omega_{M} /(N-1), 2 \omega_{M}\right]$, which we refer as linear distribution, for an impulsive excitation of the master, where $\dot{x}_{N}(0)=1 \mathrm{~m} / \mathrm{s}$, and with the following parameters $N=300, M=1 \mathrm{~kg}, \varepsilon=m(N-1) / M=0.1$ which is the mass ratio, $\omega_{M}=1 \mathrm{rad} / \mathrm{s}$. It appears more than 85 percent of the energy remain stored within the satellite structure.

\subsection{Comparison of linear and nonlinear attachments}

To check the effectiveness of the CoAt, the previous results are compared with that of a linear oscillator coupled to a NES [15]. The system consists of a linear oscillator, of mass $m_{1}$ and stiffness $k_{1}$, coupled through an essentially nonlinear stiffness $k_{2}$ to a mass $m_{2}$, the equations of motion are

$$
\left\{\begin{array}{l}
\ddot{x}_{1}+\omega_{1}^{2} x_{1}+\lambda_{1} \dot{x}_{1}+\lambda_{2}\left(\dot{x}_{1}-\dot{x}_{2}\right)+\omega_{2}^{2}\left(x_{1}-x_{2}\right)^{3}=0 \\
\varepsilon \ddot{x}_{2}-\lambda_{2}\left(\dot{x}_{1}-\dot{x}_{2}\right)-\omega_{2}^{2}\left(x_{1}-x_{2}\right)^{3}=0
\end{array}\right.
$$

where $\omega_{1}=\sqrt{k_{1} / m_{1}}, \omega_{2}=\sqrt{k_{2} / m_{1}}, \varepsilon=m_{2} / m_{1}, \lambda_{1}=c_{1} / m_{1}, \lambda_{2}=c_{2} / m_{1}$, with $c_{1}$ and $c_{2}$ the damping coefficient. As a first example, Fig. 4 shows the exchanges of energy after the impulsive excitation of the undamped linear oscillator, with the following parameters: $\dot{x}_{1}(0)=1 \mathrm{~m} / \mathrm{s}, \varepsilon=0.1$, and $\omega_{1}=\omega_{2}=1 \mathrm{rad} / \mathrm{s}$. In this case the energy is periodically transferred back and forth from the primary oscillator. This result is not surprising, since Lee et al. [15] demonstrated that a nonlinear beat phenomenon takes place between the linear oscillator and the NES. As a second example, the same system is studied with light damping, $\lambda_{1}=\lambda_{2}=0.0015$. Fig. 5 shows how the undamped response of the master decays much faster than the one of the damped linear oscillator with the NES.

These simple but significant examples enlighten the key role played by (i) the special architecture and (ii) the complexity of the system in producing an effective energy pumping. Eq. (7), which is indeed the fruit of the way the CoAt is connected to the master, shows that the more complex the system is, the faster the loss of coherence among the modal components, the smaller the mean value and the variance of the energy are. The nonlinearity alone does not provide better results than those provided by a properly designed CoAt. Indeed, Nekhoroshev's or KAM theorems explain that the motion of a weakly nonlinear system remains very close to the one of the linearized system so, if the system has a few degrees of freedom, its

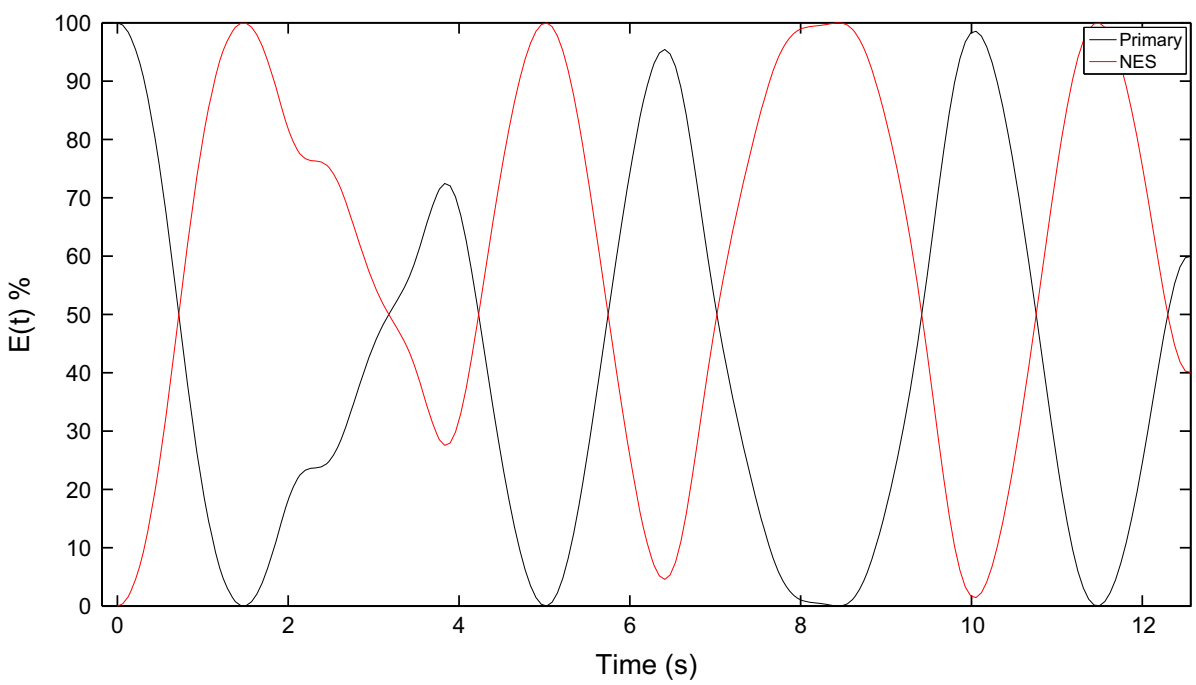

Fig. 4. Percentage of the instantaneous energy in the linear oscillator and the NES. 


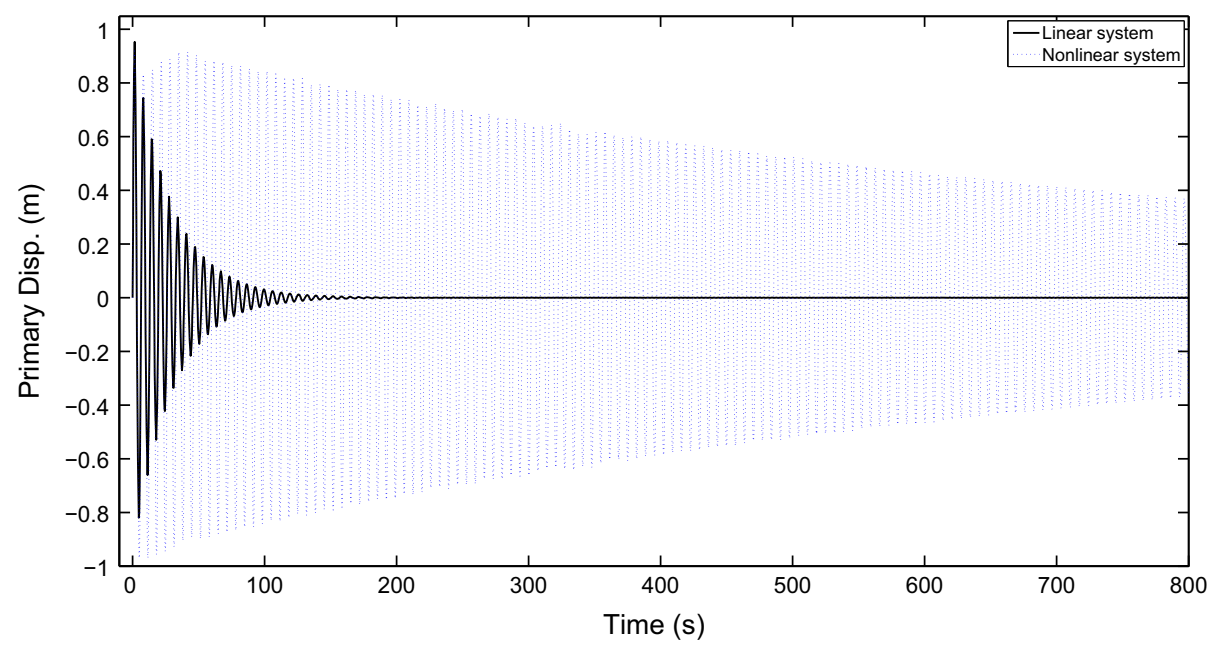

Fig. 5. Displacement of primary structures coupled to a LES and to a NES in continuous and dashed line, respectively.

almost periodic motion is driven by a few, detectable, frequencies. The vast existing literature on energy pumping [1-16] relies on the adoption of strong nonlinear connections between the master and the satellite, considering architectures with a few degrees of freedom only. As a result, energy pumping activates only above a specific energy threshold, a proper level of damping is required and/or the attached satellite has to have a large mass.

In this context, the multi-degrees of freedom NES studied in [52] needs a mention. The considered system is a two degrees of freedom (dof) linear primary oscillator connected through a weak linear stiffness to a 3-dof nonlinear attachment possessing essential stiffness nonlinearities. One of the main results of this architecture is the ability of the multi-dof NES to trap a relevant part of the injected energy for low values of the applied impulse: a goal that cannot be achieved employing a single-dof NES [10]. However, the use of the CoAt presents some advantages over the multi-dof NES: (i) it provides a faster energy transfer and (ii) while the whole mass of the CoAt is a small fraction that of the master, the total mass of the multidof NES is 3 times the one of the primary. On the other hand, the positive element of the NES is that, in general, it requires a smaller number of degrees of freedom.

\section{Energy pumping in linear systems with a complex attachment}

\subsection{Master response decomposition}

Eq. (7) only provides information regarding the average motion and does not prevent localized (in time) energy returns to the master, which are caused by constructive interference among the modal components in Eqs. (3) and (6). In order to get insight into the energy sharing process, interaction of modal component over time is developed next.

For a system equipped with a linear frequency distribution within the attachment, the spectrum of the coefficients $a_{j}$ and of the modal energies $E_{j}$ are nearly symmetric with respect to the mode index $j_{0}$ [35], slightly smaller than $N / 2$, as depicted in Fig. 6 for $N=300$ and $j_{0}=144$.

Then

$$
a_{j_{0}+j} \approx a_{j_{0}-j+1}, j=1,2, . j_{0}
$$

and Eq. (3) is rearranged as follows:

$$
x_{N}(t)=\left[\sum_{j^{-}=1}^{j_{0}} a_{j^{-}} \sin \left(\omega_{j}-t\right)+\sum_{j^{+}=j_{0}+1}^{2 j_{0}} a_{j^{+}} \sin \left(\omega_{j^{+}} t\right)\right]+\sum_{j=2 j_{0}+1}^{N} a_{j} \sin \left(\omega_{j} t\right)
$$

using Eq. (9), the previous equation can be approximated as

$$
x_{N}(t) \approx\left[\sum_{j^{-}=1}^{j_{0}} a_{j^{-}}\left(\sin \left(\omega_{j^{-}} t\right)+\sin \left(\omega_{j^{+}} t\right)\right)\right]+\sum_{j=2 j_{0}+1}^{N} a_{j} \sin \left(\omega_{j} t\right)
$$

then, the prosthaphaeresis formulas lead to

$$
x_{N}(t) \approx\left[\sum_{j=1}^{j_{0}} 2 c_{j} \cos \left(\frac{\omega_{j^{+}}-\omega_{j^{-}}}{2} t\right) \sin \left(\frac{\omega_{j^{+}}+\omega_{j^{-}}}{2} t\right)\right]+\sum_{j=2 j_{0}+1}^{N} a_{j} \sin \left(\omega_{j} t\right)
$$

where $c_{j}=a_{j_{0}-j+1}, j^{-}=j_{0}-j+1, j^{-} \in\left[1, j_{0}\right]$ and $j^{+}=j_{0}+j, j^{+} \in\left[j_{0}+1,2 j_{0}\right]$. Considering $\left(\omega_{j^{+}}+\omega_{j^{-}}\right) / 2 \approx \omega_{M}$ and $\sum_{j=}$ $2 j_{0}+1^{N} a_{j} \sin \left(\omega_{j} t\right) \approx a_{\mathrm{HF}} \sin \left(\omega_{\mathrm{HF}} t\right)$, with $\quad a_{\mathrm{HF}}=\sum_{j=2 j_{0}+1}^{N} a_{j} \quad$ and $\quad \omega_{\mathrm{HF}}=\sum_{j=2 j_{0}+1}^{N} \omega_{j} /\left(N-2 j_{0}\right) \approx 2 \omega_{M}$, and setting 


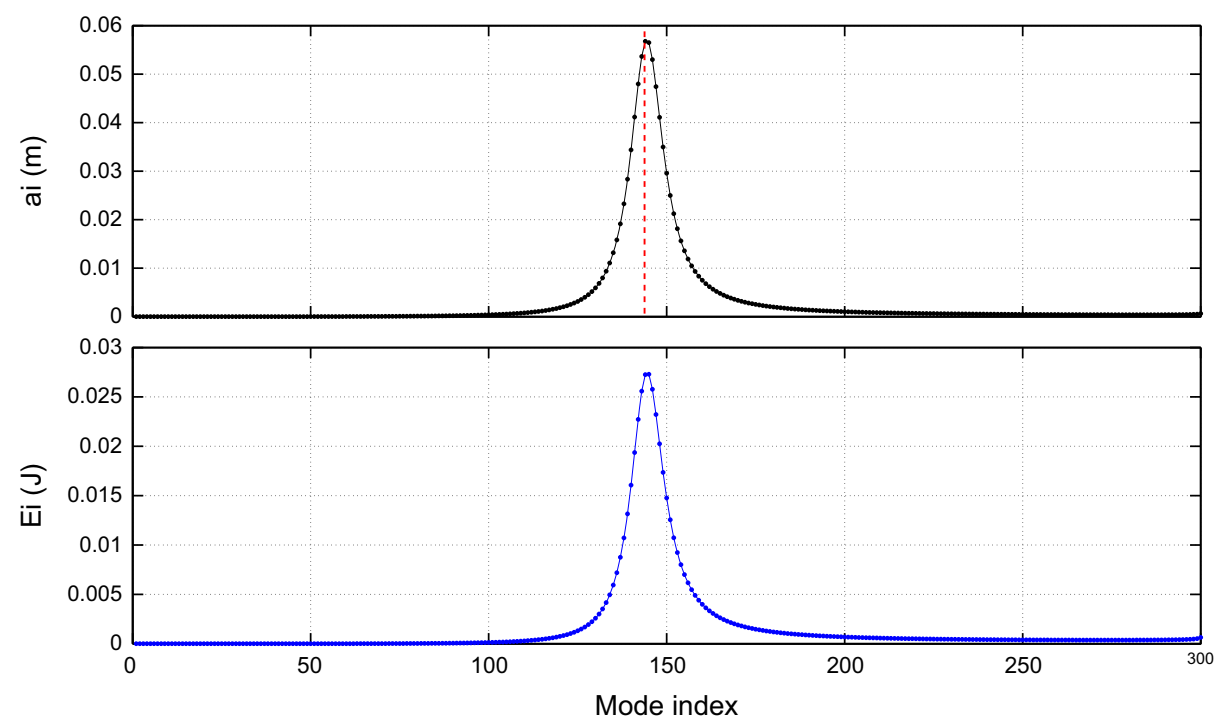

Fig. 6. Starting from the subplot on top, amplitude and modal energy spectra plotted versus the mode index $j$; the symmetry axis is depicted in dashed line.

$\Delta \omega_{j}=\left(\omega_{j^{+}}-\omega_{j^{-}}\right) / 2$, Eq. (3) is approximated as the sum of two terms:

$$
x_{N}(t) \approx x_{\text {beat }}(t)+x_{\mathrm{HF}}(t)
$$

where

- $x_{\text {beat }}(t)=\sum_{j=1}^{j_{0}} 2 c_{j} \cos \left(\Delta \omega_{j} t\right) \sin \left(\omega_{M} t\right)$ is the sum of the beat components, that has the same carrier wave $\sin \left(\omega_{M} t\right)$ while modulated by different envelopes $2 c_{j} \cos \left(\Delta \omega_{j} t\right)$;

- $x_{\mathrm{HF}}(t)=a_{\mathrm{HF}} \sin \left(2 \omega_{\mathrm{M}} t\right)$ is a low energy-high-frequency wave that vibrates at twice the master frequency.

Hilbert transform of Eq. (10) allows evaluation of the analytic signal and the instantaneous amplitude and phase, yielding

$$
\hat{x}_{N}(t)=H(t)\left(A(t) \mathrm{e}^{i \omega_{\mathrm{M}} t}+a_{\mathrm{HF}} \mathrm{e}^{i 2 \omega_{\mathrm{M}} t}\right)
$$

where the modulation theorem [39] has been used, $H(t)$ is the Heaviside step function, $i$ the imaginary unit, and

$$
A(t)=\sum_{j=1}^{j_{0}} 2 c_{j} \cos \left(\Delta \omega_{j} t\right)
$$

is the envelope of $x_{\text {beat }}(t)$. Expressing Eq. (11) in the polar form:

$$
\hat{x}_{N}(t)=X(t) \mathrm{e}^{i \varphi(t) t}
$$

where

$$
\begin{gathered}
X(t)=H(t) \sqrt{A^{2}(t)+a_{\mathrm{HF}}^{2}+2 A(t) a_{\mathrm{HF}} \cos \left(\omega_{M} t\right)} \\
\varphi(t)=\frac{A(t) \sin \left(\omega_{M} t\right)+a_{\mathrm{HF}} \sin \left(2 \omega_{M} t\right)}{A(t) \cos \left(\omega_{\mathrm{M}} t\right)+a_{\mathrm{HF}} \cos \left(2 \omega_{M} t\right)}
\end{gathered}
$$

together form the canonical pair associated with $\hat{x}_{N}(t)$. The instantaneous frequency is defined as the time derivative of the phase function $\varphi(t)$ :

$$
\omega(t)=X^{-2}(t)\left(A^{2}(t) \omega_{M}+a_{\mathrm{HF}}^{2} 2 \omega_{M}-a_{\mathrm{HF}} \dot{A}(t) \sin \left(\omega_{M} t\right)+3 A(t) a_{\mathrm{HF}} \omega_{M} \cos \left(\omega_{\mathrm{M}} t\right)\right)
$$

where $\dot{A}(t)$ is $\mathrm{d} A(t) / \mathrm{d} t$. Eqs. (10), (11), (14), and (15) suggest that

i. as the interference between the beats is constructive $A(t)>>a_{\mathrm{HF}}$ thus $X(t) \approx H(t) A(t)$ and $\omega(t) \approx \omega_{M}$ : the frequency of motion of the primary is not influenced by the satellite structure, while its amplitude is controlled by the envelope of $x_{\text {beat }}(t)$;

ii. when the interference among the beats is destructive, $A(t) \approx 0$, thus $X(t) \approx H(t) a_{\mathrm{HF}}$ and $\omega(t) \approx 2 \omega_{M}$ : the frequency of motion of the master is twice its uncoupled frequency, its vibrations are controlled by the low energy, high-frequency, modal components; and 
iii. if $A(t)$ undergoes a rapid variation, the term controlled by $\dot{A}(t)$ introduces a frequency modulation around the carrier frequency.

It appears that the envelope of $x_{\text {beat }}(t)$ has a fundamental role on the dynamics of the energy pumping. The time evolution of $A(t)$ is indeed analysed in the next section.

\subsection{Frequency intermittency effects}

It can be shown that the eigenfrequencies of the coupled system nearly coincide with the corresponding uncoupled frequencies [26]. Thus for a linear frequency distribution within the attachment, the uncoupled frequencies of the satellite structure are $\omega_{0 i}=i 2 \omega_{M} /(N-1), i=1,2, \ldots, N-1$, and the eigenfrequencies are approximated as follows:

$$
\omega_{s} \approx s 2 \omega_{M} / N, \quad s=1,2, \ldots, N
$$

Using Eq. (16) with Eq. (12), $\Delta \omega_{j}$ becomes:

$$
\Delta \omega_{j}=0.5\left(\omega_{j^{+}}-\omega_{j^{-}}\right) \approx(2 j-1) \omega_{M} / N, \quad j=1,2, \ldots, j_{0}
$$

The frequencies in Eq. (12) are roughly odd multiples of the fundamental frequency $\Omega=\Delta \omega_{1} \approx \omega_{M} / N$. Through Eq. (17), Eq. (12) becomes a summation of harmonic functions:

$$
A(t) \approx \sum_{s=1}^{j_{0}} 2 c_{s} \cos \left(s_{\text {odd }} \Omega t\right)
$$

with $s_{\text {odd }}=2 s-1$. Since $c_{s} / c_{s-1} \approx \alpha \rightarrow c_{s} \approx \alpha^{S-1} c_{1}$, with $\alpha<1$, and using Euler's formula Eq. (18) is

$$
A(t) \approx \sum_{s=1}^{j_{0}} c_{s}\left(\mathrm{e}^{i(2 s-1) \Omega t}+\mathrm{e}^{-i(2 s-1) \Omega t}\right)
$$

and then

$$
A(t) \approx \frac{c_{1}}{\alpha}\left[\mathrm{e}^{-i \Omega t}\left(\sum_{s=0}^{j_{0}}\left(\alpha \mathrm{e}^{i 2 \Omega t}\right)^{s}-1\right)+\mathrm{e}^{i \Omega t}\left(\sum_{s=0}^{j_{0}}\left(\alpha \mathrm{e}^{-i 2 \Omega t}\right)^{s}-1\right)\right]
$$

Both summations are geometric series, thus

$$
A(t) \approx \Delta a \cos (\Omega t) /(1-\cos (2 \Omega t))
$$

where $\Delta a=a_{j_{0}}-a_{j_{0}-1}$ is the amplitude difference between the two most energetic modes. Each summation in Eq. (19) is reminiscent of a Dirichlet kernel, a periodic and rapidly changing function that gives Eq. (20) the following properties:

- $A(t)$ is approximated by a periodic function of period $T_{\text {beat }}=\pi / \Omega$;

- the approximated expression of $A(t)$ has a sharp decay around the extrema located at $0, T_{\text {beat }}, 2 T_{\text {beat }}, \ldots$, and decays as $1 /(2 \Omega t)^{2}$.

In light of the previous results, the instantaneous frequency of the primary is expected to display intermittency between $\varpi_{M}$ and $2 \varpi_{M}$. Eq. (20) shows that the beat components have constructive interference in the neighborhoods of $0, T_{\text {beat }}, 2 T_{\text {beat }}, \ldots$, thus $A(t)>>a_{\mathrm{HF}}$ and Eq. (15) is dominated by $\varpi_{M}$. Outside these neighborhoods, the beat components have destructive interference and $A(t) \approx 0$, and Eq. (15) is then controlled by the high frequency component $2 \varpi_{M}$. It is worth underlining that the frequency jump, from $\varpi_{M}$ to $2 \varpi_{M}$, of the primary structure, is quite surprising, particularly considering the entire system is linear and autonomous. In fact, for a linear system the linear attachment does not act with only a purely damping effect [31], but produces frequency switching as shown, similar to frequency jumps observed when using a nonlinear attachment [15].

\subsection{Numerical experiments and discussion}

The findings of the previous section are here validated using empirical mode decomposition and normalized Hilbert transform, which provide the empirical evidence of the existence of intermittent frequency.

Fig. 7 shows the envelope defined by Eq. (12), observations about the accuracy of Eq. (20) follow:

- Eq. (20) provides qualitative information at the early stage of motion, since $A(t)$ has sharp peaks in the neighborhood of $0, T_{\text {beat }}, 2 T_{\text {beat }}, \ldots$, while it approaches zero outside these neighborhoods;

- with increasing time, Eq. (20) becomes less accurate, since the extrema of $A(t)$ become less sharp, while more and more ripples precede each extremum, as a consequence the time intervals where $A(t) \approx 0$ progressively reduce to zero.

The inadequacy of Eq. (20) to estimate $A(t)$ for long times is a consequence of the harmonic approximation (17). More precisely, the small errors committed in Eq. (17), involving the eigenfrequencies, are negligible at early stage, but progressively destroy the 


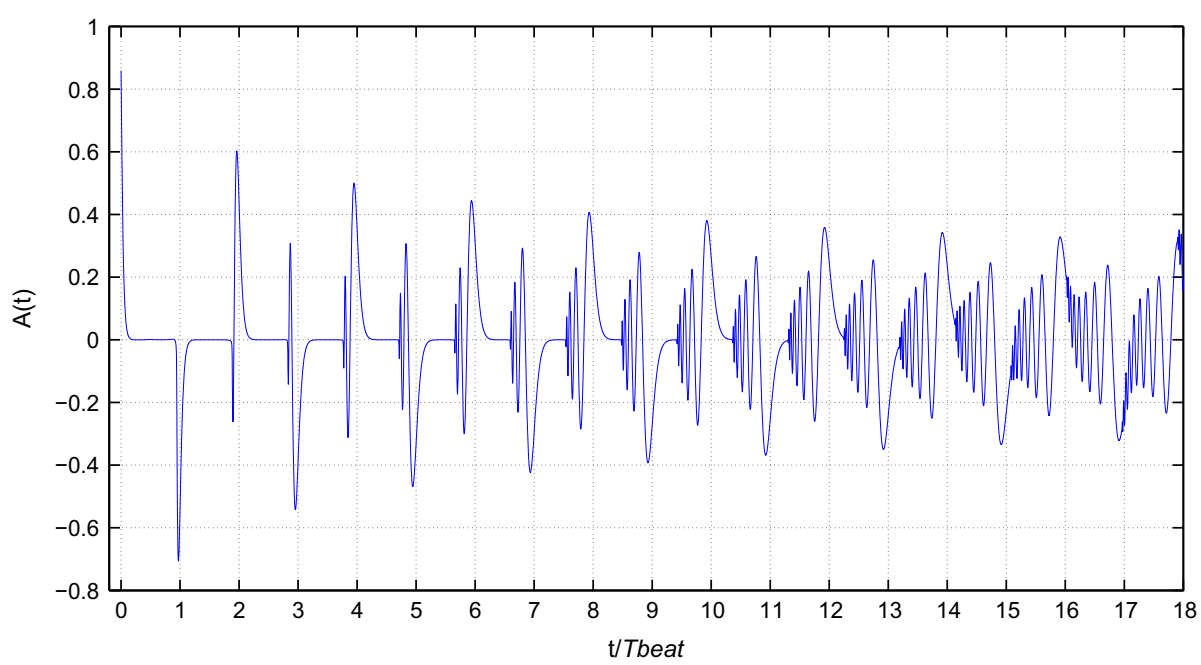

Fig. 7. The envelope $A(t)$ plotted versus the normalized time $t / T_{\text {beat }}$.
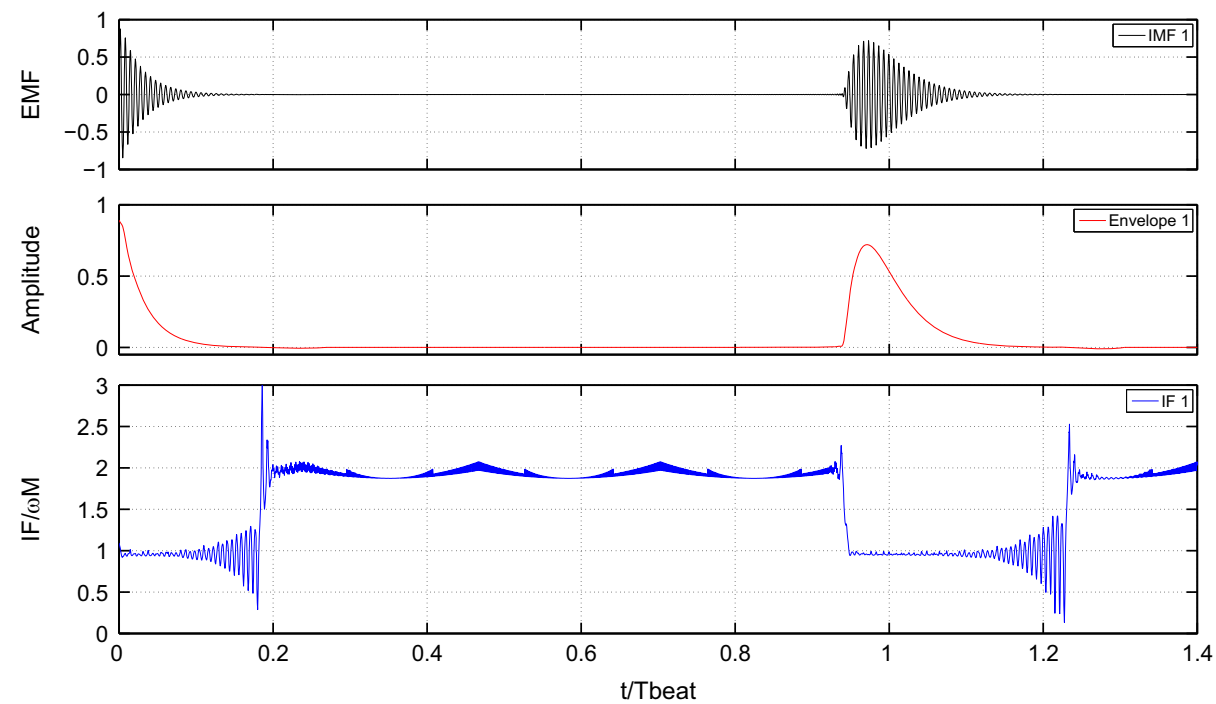

Fig. 8. Starting from the subplot on top, the first IMF of the master displacement, the instantaneous amplitude and normalized frequency, plotted versus the normalized time $t / T_{\text {beat }}$.

strict periodicity of motion predicted by Eq. (20). This suggests that a transition in the nature of motion is taking place: a more accurate model is necessary to describe the interaction of the modal components after the early stage of motion.

To verify the existence of an intermittent frequency and more generally to get insightful information concerning the energy sharing process, the transient response of the master is processed with the empirical mode decomposition (EMD) [38,42] and the normalized Hilbert transform (NHT) [41]. EMD is a well established method that can be used to decompose a signal in terms of monocomponent functions called intrinsic mode functions (IMFs), which can be thought as intrinsic oscillatory modes imbedded in the data. NHT is an empirical technique designed to separate the AM and FM parts of each IMF. It allows evaluating the instantaneous frequency (IF) of the IMF without the use of the Hilbert transform (HT), since, as pointed out in [41], direct application of the HT is often problematic and may produce meaningless results. After the master response has been processed via EMD, only two IMFs are generated and since the energy of the second IMF is much smaller than the first one (roughly 1/100), only the first IMF is considered. This result confirms the assumption made in Section 3.1, where the master response was assumed monocomponent.

Fig. 8 shows the first IMF, its instantaneous amplitude and frequency obtained with NHT. Numerical results are in good agreement with predictions made by Eqs. (15) and (20):

- there exists an initial transient where the master has a damped response and the envelope has a sharp decay, followed by a stationary state where its value is approximately zero until $t \approx T_{\text {beat }}$ when the envelope regains a sharp crest and most of the energy is returned to the master; 
- the instantaneous frequency is initially equal to $\omega_{M}$, due to the rapid variation of the envelope, the term controlled by $\dot{A}(t)$ introduces a frequency modulation and causes higher and higher fluctuations around the carrier frequency $\varpi_{M}$, as described by the sinusoidal term in Eq. (15) and as discussed in [38];

- there is a frequency jump from $\varpi_{M}$ to $2 \varpi_{M}$ as the instantaneous amplitude reaches the trigger value at $t / T_{\text {beat }} \approx 0.18$. Fig. 9 shows a close-up of the master response during the time interval $t / T_{\text {beat }}=[0.15,0.3]$ : the frequency doubling is clear;

- corresponding to the sharp rise of the envelope, at $t / T_{\text {beat }} \approx 0.95$, a backward frequency jump, from $2 \varpi_{M}$ to $\varpi_{M}$, takes place.

It is worth mentioning that the third subplot in Fig. 8 is qualitatively similar to the instantaneous frequency obtained by Starosvetsky and Gendelman [50] with the Hilbert vibration decomposition, studying the dynamics of 2dof linear subsystem with close frequencies attached with a NES.

Fig. 10 shows a frequency-energy plot of $E_{N}(I F(t))$, within the time interval $t / T_{\text {beat }}=[0,0.97]$; at $t / T_{\text {beat }}=0.97$ the energy returned to the master has a maximum. The graph is in the form of a loop, sketched in dashed line in figure:

- there exists an initial transient where the primary is in resonance with the most energetic resonators, during this phase the energy is almost monotonically transferred from the master to the satellite structure. The frequency of motion is

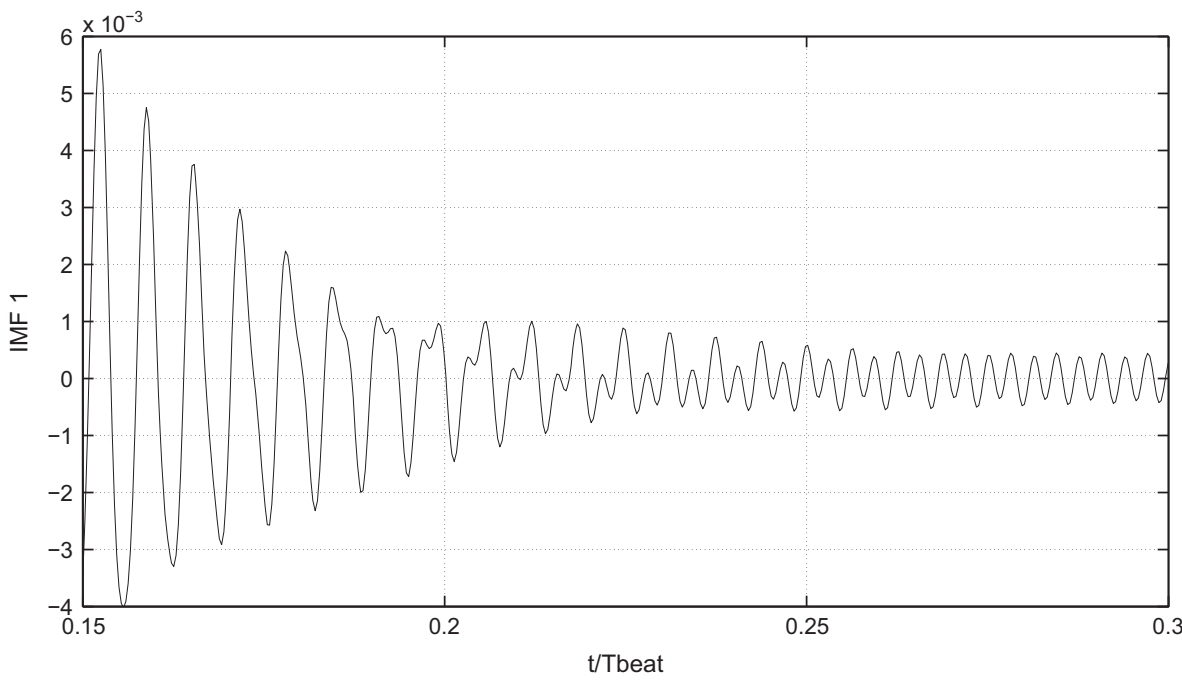

Fig. 9. Close-up of the first IMF plotted versus the normalized time $t / T_{\text {beat }}$.

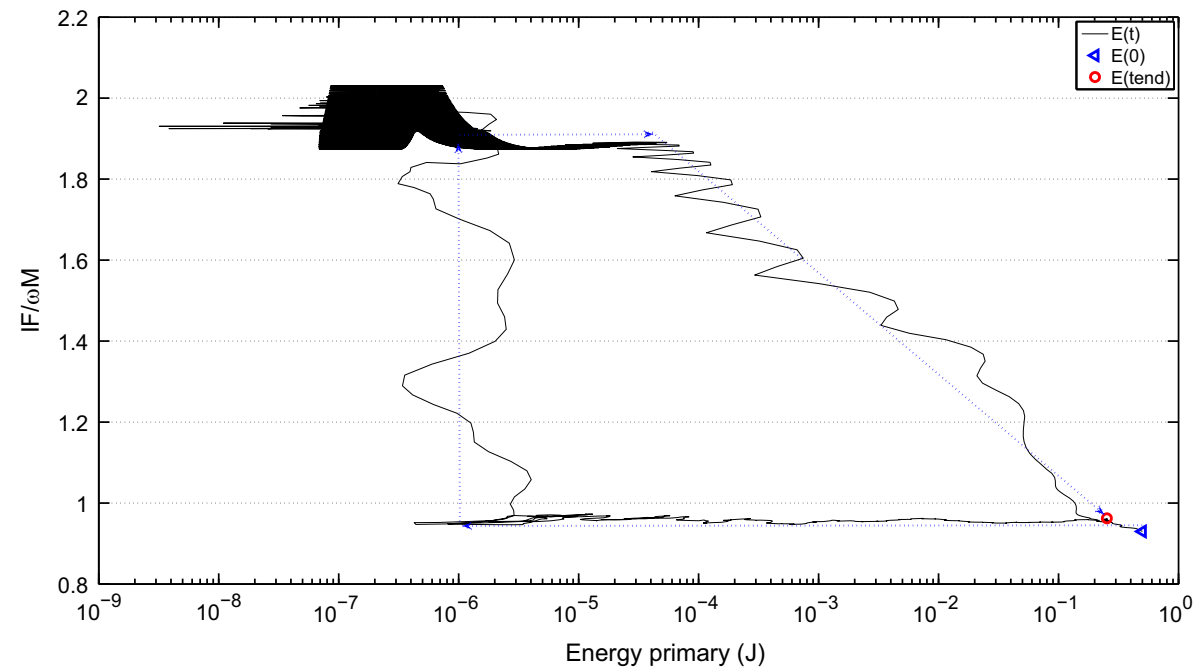

Fig. 10. Frequency-energy plot related to the master, i.e. $E_{N}(I F(t))$; along the $x$ and $y$-axis the energy of the primary and the normalized IF, respectively. 

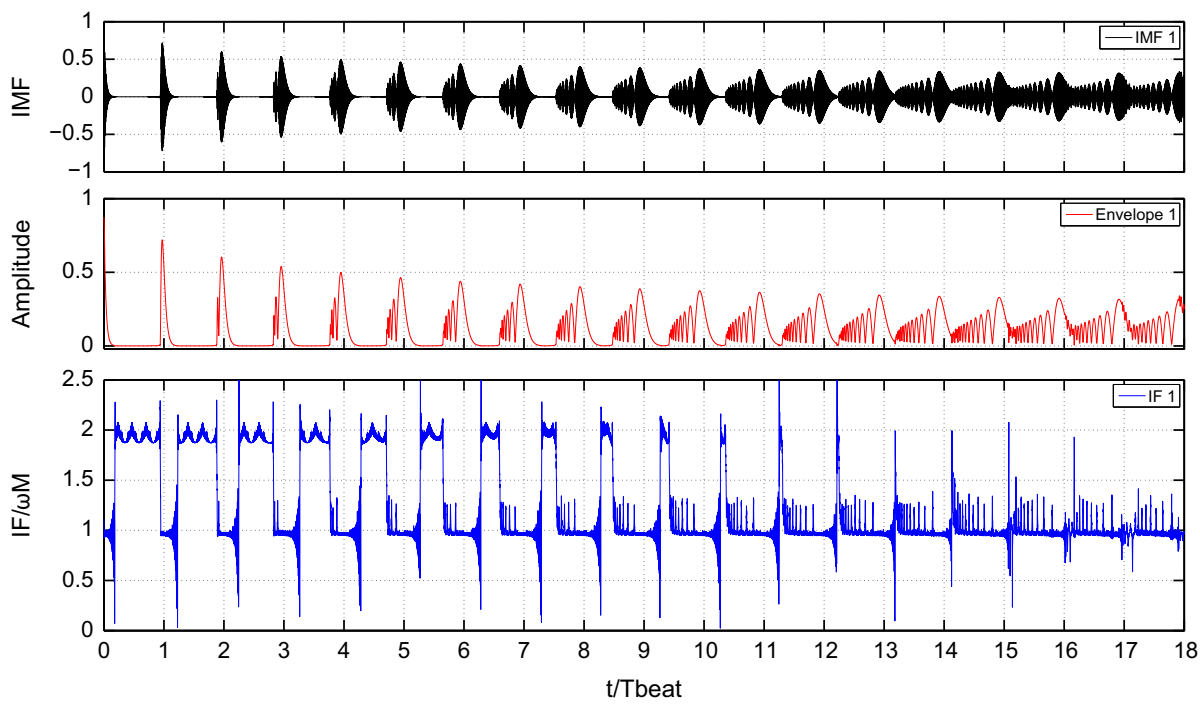

Fig. 11. Starting from the subplot on top, the first IMF, the instantaneous amplitude and frequency related to the master displacement versus the normalized time $t / T_{\text {beat }}$.

roughly equal to $\varpi_{M}$ and the satellite acts as a damper. The numerical IF is slightly smaller than $\varpi_{M}$ because, as pointed out in Section 3.1, $j_{0}$ is slightly smaller than $N / 2$ then $0.5\left(\omega_{j^{+}}+\omega_{j^{-}}\right)<\omega_{M}$;

- as nearly the entire energy is transferred to the satellite, the IF experiences a sudden jump, from $\varpi_{M}$ to $2 \varpi_{M}$. The primary and the most energetic resonators remain energetically decoupled and, as a consequence, the energy is trapped within the satellite structure. The master is now in resonance with a few, low energy, high-frequency resonators, and exchanges energy with them in a reversible manner;

- as the master energy reaches the trigger value, roughly $10^{-4} \mathrm{~J}$, a backward jump takes place, which brings the IF back to $\varpi_{M}$; during this phase most of the energy is rapidly returned to the master;

- the end point of the loop does not coincide with the starting point, showing there is a part of the energy which remains irreversibly trapped in the satellite structure.

The IMF and the IF of the master response for $t>T_{\text {beat }}$ are plotted in Fig. 11. The figure highlights the existence of a mesoscale process where the time response of the master changes from a nearly periodic, where most of the energy is periodically transferred to and from the master with period $T_{\text {beat }}$, to a stationary state, where most of the energy is permanently stored into the satellite structure. During this mesoscale process two different alternating, or intermittent, phases are identified, corroborating the prediction made in Section 3.1:

i. one governs the energy transfer to and from the master and is characterized by an IF roughly equal to $\varpi_{M}$;

ii. the other one is characterized by $\mathrm{IF} \approx 2 \omega_{M}$, the primary and the most energetic resonators are energetically decoupled and the energy is reversibly exchanged between the master and a few resonators at high frequency.

iii. The time durations of these alternating phases change with time. Indicating with $\Delta t_{\omega_{M}}$ and $\Delta t_{2 \omega_{M}}$ the nondimensional time durations, normalized with $T_{\text {beat }}$, at the beginning $\Delta t_{2 \omega_{M}} \gg \Delta t_{\omega_{M}}$, as the time spends $\Delta t_{2 \omega_{M}} \rightarrow 0$ and $\Delta t_{\omega_{M}} \rightarrow 1$.

iv. The mesoscale duration $T_{\text {mes }}$ is assumed to be the time when $\Delta t_{2 \omega_{M}}$ becomes zero: for $t>T_{\text {mes }}$ no more intermittency is observed and the IF oscillates around the uncoupled frequency of the master.

The sharp peaks in the IF plot, observed after $\Delta t_{2 \omega_{M}}=0$, are errors near the neighborhood of minimum amplitude locations, that arise due to the violation of the Bedrosian theorem [39,41].

The points (i)-(iv) are strictly related to the time evolution of the envelope $A(t)$, previously discussed and highlighted in Fig. 7. It may be suspiring that for $t>T_{\text {mes }}$ no energy return is observed, even if the master remains in resonance with the most energetic resonators. The energy is reversibly exchanged, but its amplitude remains relatively small, close to the mean value $\left[E_{N}\right]$. An intuitive explanation for that can be found by considering the master velocity or energy expressions, Eqs. (3) and (5). For $t>0$, the coherence among the modal components is progressively lost and each one contributes to the master energy with a fraction of its modal energy: a vast energy return is possible only if the initial coherence is regained. The numerical experiments have yielded two characteristic time scales: (i) for $t<T_{\text {mes }}$ the coherence among the modal components is regained roughly at each $T_{\text {beat }}$ period, thus the master response has a clear almost periodic evolution, and (ii) for $t>T_{\text {mes }}$ the phases of the modal components are uncorrelated. It follows that the resultant force applied from the satellite has a small amplitude, even if the energy is almost completely stored into the CoAt, thus the motion of the master is roughly harmonic with frequency $\varpi_{M}$ and small amplitude. 

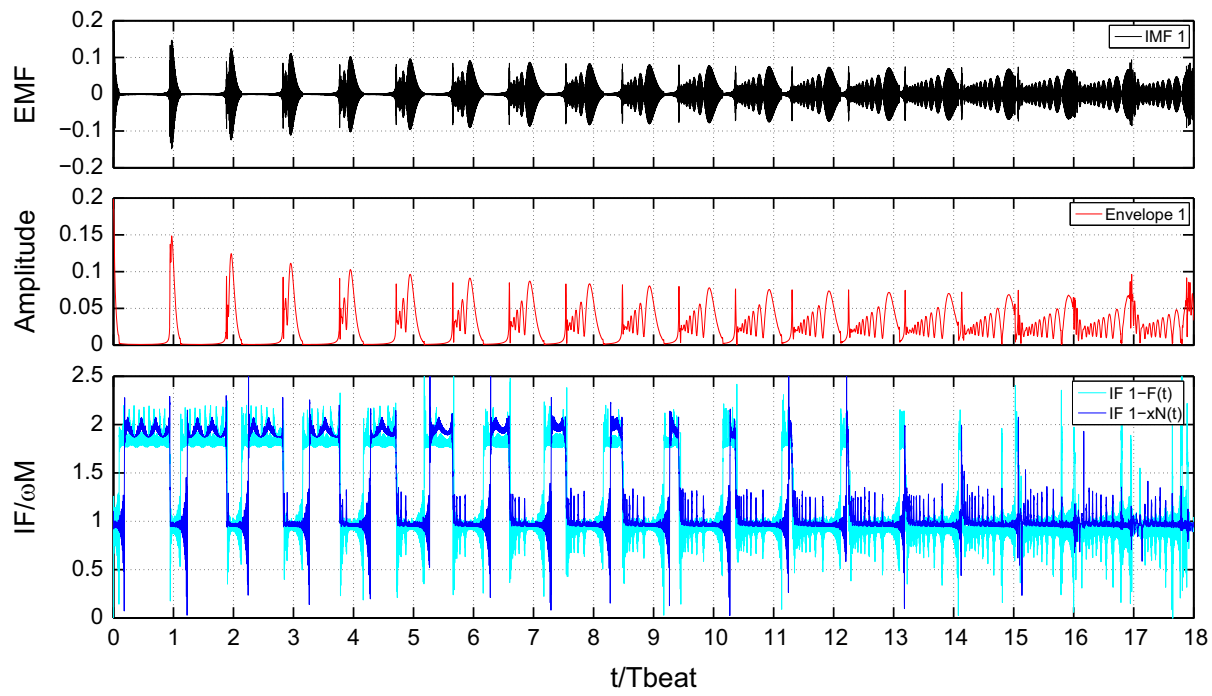

Fig. 12. Starting from the subplot on top, the first IMF, instantaneous amplitude and frequency (cyan) related to $F(t)$ versus the normalized time $t / T_{\text {beat }}$.

Finally, we examine the net force $F(t)$ exerted from the satellite to the master. Eq. (1) is rearranged as follows:

$$
\ddot{x}_{N}(t)+\left(\omega_{M}^{2}+\alpha \sum_{j=1}^{N-1} \omega_{0 j}^{2}\right) x_{N}(t)=\alpha \sum_{j=1}^{N-1} \omega_{0 j}^{2} x_{j}(t)
$$

where $\alpha=m / M$, thus

$$
F(t)=\alpha \sum_{j=1}^{N-1} \omega_{0 j}^{2} x_{j}(t)
$$

When EMD is applied to $F(t)$, only one significant IMF is generated and the instantaneous amplitude and frequency are obtained with the NHT, plotted in Fig. 12 for $t=\left[0,18 T_{\text {beat }}\right]$. The time histories related to $F(t)$ are qualitatively similar to the ones of $x_{N}(t)$, only the forward frequency jumps, from $\varpi_{M}$ to $2 \varpi_{M}$, are slightly anticipated for $F(t)$; thus $x_{N}(t)$ remains longer and longer in resonance with $F(t)$, being the corresponding instantaneous amplitudes and frequencies strictly correlated. It arises one of the main advantages of using a LES, which allows keeping the amplitude of the force exchanged with the primary very small even if most of the energy is efficiently targeted into the CoAt.

\section{Conclusions}

This paper reports the realization of an effective targeted energy pumping from a linear oscillator to a set of linear oscillators, coupled to it in parallel. The existence of a multiphase process where the instantaneous frequency, associated to the master response, is characterized by intermittency and assumes alternating values between $\varpi_{M}$ and $2 \varpi_{M}$, has been unveiled. An intermittent frequency in a linear autonomous system is rather unexpected and, to the authors' knowledge, has never been reported before.

The results demonstrate the efficacy of using a CoAt as passive absorbers of broadband energy injected into the primary structure. Comparative studies, with nonlinear energy sinks, have shown this special architecture allows trapping the energy in a very efficient way. The proposed device represents indeed a novelty in the field of energy pumping, up now focused on nonlinear attachments only.

The paper also explains the mechanism behind the apparent damping phenomenon, unveiling the existence of a mesoscale, where the master response changes from almost periodic, where most of the energy is periodically transferred to and from the master with a dominant period $T_{\text {beat }}=\pi N / \varpi_{M}$, to a random noise motion, where the energy of the master remains very small, close to the mean value $E_{\text {tot }} / N$.

\section{References}

[1] H.J. Rice, J.R. McCraith, Practical non-linear vibration absorber design, Journal of Sound and Vibration 116 (3) (1987) $545-559$.

[2] M.N.S. Hadi, Y. Arfiadi, Optimum design of absorber for Ndof structures, Journal of Structural Engineering 124 (1998) $1272-1280$.

[3] M.P. Singh, L.M. Moreschi, Optimal placement of dampers for passive response control, Earthquake Engineering E Structural Dynamics 31 (2002) 955-976. 
[4] J. Shaw, S.W. Shaw, A.G. Haddow, On the response of the nonlinear vibration absorber, International Journal of Non-Linear Mechanics 24 (1989) 281-293.

[5] I.N. Jordanov, I.B. Cheshankov, Optimal design of linear and nonlinear dynamic vibration absorbers, Journal of Sound and Vibration 123 (1988) $157-170$.

[6] S. Natsiavas, Steady state oscillations and stability of non-linear dynamic vibration absorbers, Journal of Sound and Vibration 156 (2) (1992) $227-245$.

[7] O. Gendelman, L.I. Manevitch, A.F. Vakakis, R.M. Closkey, Energy pumping in nonlinear mechanical oscillators: Part I-dynamics of the underlying Hamiltonian systems, Journal of Applied Mechanics 68 (2001) 34-41.

[8] A.F. Vakakis, O. Gendelman, Energy pumping in nonlinear mechanical oscillators: Part II-resonance capture, Journal of Applied Mechanics 68 (2001) $42-48$.

[9] O.V. Gendelman, Transition of energy to a nonlinear localized mode in a highly asymmetric system of two oscillators, Nonlinear Dynamics 25 (2001) 237-253.

[10] McFarland, D.M., Bergman, L., Vakakis, A.F., Manevitch, L.I., Gendelman, O., Energy pumping into passive nonlinear energy sinks attached to forced linear substructures: Analytical and experimental results, Proceedings of the Ninth Conference on Nonlinear Vibrations, Stability, and Dynamics of Structures, Virginia, July 28-August 1, 2002, Session 3.

[11] A.F. Vakakis, L.I. Manevitch, O. Gendelman, L. Bergman, Dynamics of linear discrete systems connected to local, essentially non-linear attachments, Journal of Sound and Vibration 264 (2003) 559-577.

[12] A.F. Vakakis, Inducing passive nonlinear energy sinks in linear vibrating systems, Journal of Vibration and Acoustics 123 (3) (2001) $324-332$.

[13] E. Gourdon, C.H. Lamarque, S. Pernot, Contribution to efficiency of irreversible passive energy pumping with a strong nonlinear attachment, Nonlinear Dynamics 50 (2007) 793-808.

[14] E. Gourdon, N.A. Alexander, C.A. Taylor, C.H. Lamarque, S. Pernot, Nonlinear energy pumping under transient forcing with strongly nonlinear coupling: theoretical and experimental results, Journal of Sound and Vibration 300 (2007) 522-551.

[15] Y.S. Lee, G. Kerschen, A.F. Vakakis, P. Panagopoulos, L.A. Bergman, D.M. Mc- Farland, Complicated dynamics of a linear oscillator with an essentially nonlinear local attachment, Physica D 204 (2005) 41-69.

[16] G. Kerschen, K. Worden, A.F. Vakakis, J.C Golinval, Past, present and future of nonlinear system identification in structural dynamics, Mechanical Systems and Signal Processing 20 (505-592) (2006) 2006.

[17] A. Haro, R. Llave, New mechanisms for lack of equipartition of energy, Physical Review Letters 85 (9) (2000) 1859-1862.

[18] F. Fassò, M. Guzzo, G. Benettin, Nekhoroshev-stability of elliptic equilibria of Hamiltonian systems, Communications in Mathematical Physics 197 (1997) $347-360$.

[19] A. Giorgilli, Notes on exponential stability of Hamiltonian systems, Dynamical Systems. Part I: Hamiltonian Systems and Celestial Mechanics/[a cura di] Stefano Marmi, Scuola Normale Superiore, Pisa, 2003, pp. 87-198, ISBN 978-88-7642-294-2.

[20] J. Poschel, A lecture on the classical KAM-theorem, Proceedings of Symposia in Pure Mathematics (AMS) 69 (2001) 707-732.

[21] C. Soize, A model and numerical method in the medium frequency range for vibroacoustic predictions using the theory of structural fuzzy, Journal of Acoustical Society of America 94 (1993) 849-865.

[22] A.D. Pierce, V.W. Sparrow, D.A. Russel, Fundamental structural-acoustic idealization for structure with fuzzy internals, Journal of Vibration and Acoustics 117 (1995) 339-348.

[23] M. Strasberg, D. Feit, Vibration damping of large structures induced by attached small resonant structures, Journal of Acoustical Society of America 99 (1996) 335-344.

[24] M. Strasberg, Continuous structures as fuzzy substructures, Journal of Acoustical Society of America 100 (5) (1996) 3456-3459.

[25] K. Xu, T. Igusa, Dynamic characteristics of multiple substructures with closely spaced frequencies, Earthquake Engineering and Structural Dynamics 21 (12) (1992) 1059-1070.

[26] R.L. Weaver, The effect of an undamped finite degree of freedom 'fuzzy' substructure: numerical solution and theoretical discussion, Journal of Acoustical Society of America 101 (1996) 3159-3164.

[27] R.L. Weaver, Equipartition and mean square response in large undamped structures, Journal of Acoustical Society of America 110 (2001) 894-903.

[28] G. Maidanik, Induced damping by a nearly continuous distribution of a nearly undamped oscillators: linear analysis, Journal of Sound and Vibration 240 (2001) 717-731.

[29] R.S Langley, Can an undamped oscillator dissipate energy? Journal of Sound and Vibration 206 (1997) 624-626.

[30] C.E. Celik, A. Akay, Dissipation in solids: thermal oscillations of atoms, Journal of Acoustical Society of America 108 (2000) 184-191.

[31] A. Carcaterra, A. Akay, Transient energy exchange between a primary structure and a set of oscillators: return time and apparent damping, Journal of Acoustical Society of America 115 (2004) 683-696.

[32] A. Carcaterra, A. Akay, I.M. Koc, Near-irreversibility and damped response of a conservative linear structure with singularity points in its modal density, Journal of Acoustical Society of America 119 (2006) 2141.

[33] A. Akay, Zhaoshun Xu, A. Carcaterra, I. Murat Koç, Experiments on vibration absorption using energy sinks, Journal of Acoustical Society of America 118 (2005) 3043.

[34] I. Murat Koç, A. Carcaterra, Zhaoshun Xu, A. Akay, Energy sinks: vibration absorption by an optimal set of undamped oscillators, Journal of Acoustical Society of America 118 (2005) 3031.

[35] A. Carcaterra, A. Akay, Theoretical foundation of apparent damping and energy irreversible energy exchange in linear conservative dynamical systems, Journal of Acoustical Society of America 121 (2007) 1971-1982.

[36] N. Roveri, A. Carcaterra, A. Akay, Energy equipartition and frequency distribution in complex attachments, Journal of Acoustical Society of America 126 (2009) 122-128.

[37] A. Carcaterra, A.Akay, Patent Pending, appointed inventors property University of Rome "La Sapienza", first deposit no. RM001502005, 31.2.2005 and International Patent WO2006103291-2006-10-05.

[38] N.E. Huang, Z. Shen, S.R. Long, M.C. Wu, H.H. Shih, Q. Zheng, N. Yen, C.C. Tung, H.H. Liu, The empirical mode decomposition and the Hilbert spectrum for nonlinear and non-stationary time series analysis, Proceedings of the Royal Society of London A 454 (1998) 903-995.

[39] S. Hahn, Hilbert Transforms in Signal Processing, Artech House, Boston, 1995, 442.

[40] G. Kerschen, Y.S. Lee, A.F. Vakakis, D.M. Mc- Farland, L.A. Bergman, Irreversible passive energy transfer in coupled oscillators with essential nonlinearity, SIAM Journal on Applied Mathematics 66 (2) (2006) 648-679.

[41] N.E. Huang, Z. Wu, S.R. Long, K.C. Arnold, X. Chen, K. Blank, On the instantaneous frequency, Advances in Adaptive Data Analysis 1 (2) (2009) $177-229$.

[42] Rilling G., Flandrin P., Goncalves P., On empirical mode decompositions and its algorithms, Proceedings of IEEE-EURASIP Workshop on Nonlinear Signal and Image Processing NSIP-03, Grado, Italy, 2003.

[43] J.M. Rosenblatt, M.W. Pointwise, Ergodic theorems via harmonic analysis, in: K.E. Petersen, I.A. Salama (Eds.), Ergodic Theory and its Connections With Harmonic Analysis, Proceedings of the 1993 Alexandria Conference, Cambridge University Press, Cambridge, 1995.

[44] A. Kar, Weyl's equidistribution theorem, in: Resonance, May 2003, pp. 30-37.

[45] N. Roveri, A. Carcaterra, A. Akay, Vibration absorption using non-dissipative complex attachments with impacts and parametric stiffness, Journal of Acoustical Society of America 126 (2009) 2306-2314.

[46] H. Bohr, Almost Periodic Functions, Chelsea, New York, 1947.

[47] L. Barreira, Poincaré recurrence: old and new, in: Jean-Claude Zambrini (Ed.), XIV International Congress on Mathematical Physics, World Scientific, Lisbon, Portugal, 2006, pp. 415-422.

[48] V.I. Arnold, A. Avez, Ergodic Problems of Classical Mechanics, W.A. Benjamin, Inc., New York, 1968.

[49] J.V. Josè, E.J. Saletan., Classical Dynamics: A Contemporary Approach, Cambridge University Press, Cambridge, UK, 1998. 
[50] Y. Starosvetsky, O.V. Gendelman, Interaction of nonlinear energy sink with a two degrees of freedom linear system: Internal resonance, Journal of Sound and Vibration 329 (2010) 1836-1852.

[51] Y. Starosvetsky, O.V. Gendelman, Vibration absorption in systems with a nonlinear energy sink: nonlinear damping, Journal of Sound and Vibration 324 (2009) 916-939.

[52] Stylianos Tsakirtzis, Panagiotis N. Panagopoulos, Gaetan Kerschen, Oleg Gendelman, Alexander F. Vakakis, Lawrence A. Bergman, Complex dynamics and targeted energy transfer in linear oscillators coupled to multi-degree-of-freedom essentially nonlinear attachments, Nonlinear Dynamics 48 (3) (2007) 285-318. 